Pacific Journal of 


\title{
COMPLETELY OUTER GALOIS THEORY OF PERFECT RINGS
}

\author{
JAMES OSTERBURG
}

Let $G$ be a finite completely outer group of automorphisms of a perfect ring $R$. Let $\Delta$ be the crossed product of $R$ with $G$. Then $\Delta$ modules which are $R$ projective are $\Delta$ projective and $\Delta$ submodules which are $R$ direct summands are $\Delta$ direct summands.

Let $R$ be a ring with identity. All modules and homomorphisms are unital. Let $G=\{1, \sigma, \cdots, \tau\}$ be a finite group of automorphisms of $R$.

By $R u_{\sigma}, \sigma \in G$, we mean a bi- $R$ module where $r\left(r^{\prime} u_{\sigma}\right)=\left(r r^{\prime}\right) u_{\sigma}$ and $\left(r^{\prime} u_{\sigma}\right) r=\left(r^{\prime} r^{\sigma}\right) u_{\sigma}$ for $r, r^{\prime} \in R$ and $r^{\sigma}=\sigma(r)$. We call $G$ a completely outer group of automorphisms of $R$, if for each pair $\sigma \neq \tau$ of automorphisms in $G$, the bi- $R$ modules $R u_{\sigma}$ and $R u_{\tau}$ have no nonzero isomorphic subquotients. This notion was defined by T. Nakayama in [3, p. 203] and Y. Miyashita in [2, p. 126].

Let $S$ be the fixed ring of $R$ under $G$, i.e. $\{r \in R \mid \sigma(r)=r$ for all $\sigma \in G\}$. If $G$ is completely outer, then $R$ over $S$ is a $G$-Galois extension and the center of $R$ is the centralizer of $S$ in $R$. See [2, Proposition 6.4, p. 127]. Furthermore, if $R$ is a commutative ring and $R$ over $S$ is $G$-Galois, then $G$ is a completely outer group of automorphisms of $R$. See [2, Theorem 6.6, p. 128]. If $R$ is a simple ring and $G$ contains no inner automorphisms, then $G$ is a completely outer group of automorphisms of $R$ and conversely. See [2, Corollary, p. 128].

The crossed product $\Delta$ of $R$ with $G$ is $\Sigma_{\sigma \in G} \oplus R u_{\sigma}$ with $\left(x u_{\sigma}\right) \times$ $\left(y u_{\tau}\right)=x y^{\sigma} u_{\sigma \tau}$ for any $x$ and $y$ in $R$. We can view $R$ as a left $\Delta$ module by defining $\left(x u_{\sigma}\right) r=x r^{\sigma}$ for $x$ and $r$ in $R$. Thus $R$ is a bi $\Delta-S$ module.

We now assume $R$ is a left perfect ring. These rings were studied by $\mathrm{H}$. Bass [1].

Let $J(\Delta)$ (respectively $J(R)$ ) denote the Jaccbson radical of $\Delta$ (respectively $R$ ).

Lemma 1. $J(\Delta)=J(R) \Delta=\Delta J(R)$. Thus for any left $\Delta$ module $M, J(R) M$ is a $\Delta$ submodule of $M$.

Proof. Because $\sigma(J(R))=J(R)$ for all $\sigma \in G, \quad J(R) \Delta=$ $\Delta \cdot J(R)$. Thus for any simple, nonzero left $\Delta$ module $M, J(R) M$ is a $\Delta$ submodule. Now $M$ is a finitely generated $R$ module, since $\Delta$ is a finitely generated $R$ module. Nakayama's Lemma then shows 
$J(R) M=0 . \quad$ Since $J(R)$ annihilates every simple left $\Delta$ module, $J(R) \subseteq$ $J(\Delta)$.

Since $J(\Delta)$ is a bi- $R$ submodule of $\Delta, J(\Delta)=$ $J(\Delta) \cap R u_{1}+J(\Delta) \cap R u_{\sigma}+\cdots+J(\Delta) \cap R u_{\tau}$. See [2, Proposition 6.1, p. 126]. Let $x=\delta=r u_{\sigma} \in J(\Delta) \cap R u_{\sigma}$ for $\delta$ in $J(\Delta)$ and $r \in R$. Assume $\delta=\Sigma_{\sigma \in G} y_{\sigma} u_{\sigma}, \quad y_{\sigma} \in R$, then $x=y_{\sigma} u_{\sigma}=r u_{\sigma}, \quad$ so $r u_{1}=y_{\sigma} u_{1}=$ $x u_{\sigma^{-1}} \in J(\Delta) \cap R u_{1} . \quad$ It follows that $1-y_{\sigma} s$ is right invertible in $R$ for all $s$ in $R$; since $u_{1}-y_{\sigma} s u_{1}$ in $J(\Delta)$ all $s$ in $R$. Thus $y_{\sigma} \in J(R)$; hence $x=y_{\sigma} u_{\sigma} \in J(R) u_{\sigma}$. Therefore $J(R) \Delta=J(\Delta)$.

Remark. Lemma 1 is true even if $R$ is not left perfect.

LEMMA 2. As a right $S$ module, $S$ is a direct summand of $R_{S}$. Let $J(S)$ (respectively $J(R)$ ) denote the Jacobson radical of $S$ (respectively of $R)$, then $J(S)=S \cap J(R)$ and $J(R)=J(S) R=R J(S)$. Thus if $M$ is a left $\Delta(R)$ module $J(S) M$ is a left $\Delta(R)$ submodule.

Proof. Let $\bar{\Delta}=\Delta / J(\Delta)$ and $\bar{R}=R / J(R)$. Because $R$ is perfect, $\bar{R}$ is a semisimple, Artinian ring, which makes $\bar{\Delta}$ a semisimple, Artinian ring. Thus $\bar{R}$ is a finitely generated, projective $\bar{\Delta}$ module. By the Dual Basis Lemma, there exists $f_{1}, \cdots, f_{n} \in \operatorname{Hom}_{\Delta}(\bar{R}, \bar{\Delta})$ and $\bar{x}_{1}, \cdots, \bar{x}_{n} \in$ $\bar{R}$ such that $\bar{x}=\sum_{i=1}^{n} f_{i}(\bar{x}) \bar{x}_{i}$. Since $\operatorname{Hom}_{\bar{\Delta}}(\bar{R}, \bar{\Delta}) \subseteq \operatorname{Hom}_{\bar{R}}(\bar{R}, \bar{\Delta})=\bar{\Delta}$ we conclude $\operatorname{Hom}_{\bar{\Delta}}(\bar{R}, \bar{\Delta})=\sum_{\sigma \in G} u_{\sigma} \bar{R}$. Thus each $f_{i}, i=1, \cdots, n$, is of the form $\sum_{\sigma \in G} u_{\sigma} \bar{r}_{i}$, for some suitable $\bar{r}_{i} \in R$. Let $\bar{x} \in \bar{R}$, then $\bar{x}=\sum_{i=1}^{n} f_{i}(\bar{x}) \bar{x}_{i}=$ $\sum_{i=1}^{n}\left(\sum_{\sigma \in G} \bar{x} u_{\sigma} \bar{r}_{i}\right) \bar{x}_{i}=\bar{x} \sum_{i=1}^{n} \sum_{\sigma \in G}\left(\bar{r}_{i} \bar{x}_{i}\right)^{\sigma}$. Thus $\overline{1}=\sum_{\sigma \in G} \sum_{i=1}^{n}\left(\bar{r}_{i} \bar{x}_{i}\right)^{\sigma}$. Let $\bar{d}=\sum_{i=1}^{n} \bar{r}_{i} x_{i}$, then $\operatorname{tr} \bar{d}=\overline{1}$; hence $\operatorname{tr} d-1 \in J(R) \cap S$.

Now $J(R) \cap S \subseteq J(S)$, for let $x=j=s, j \in J(R)$ and $s \in S$, then 1 - sy is right invertible in $R$ for any $y$ in $S$. Assume $(1-s y) z=1$, $z \in R$, then $(1-s y) z^{\sigma}=1$ for all $\sigma \in G$; hence $z \in S$. So $1-s y$ is right invertible in $S$ for all $y$ in $S$, thus $x \in J(S)$ or $J(R) \cap S \subseteq J(S)$.

Thus $\operatorname{tr} R+J(S)=S$, so by Nakayama's Lemma $\operatorname{tr} R=S$. Thus there is a $c$ in $R$ such that $\operatorname{tr} c=1$. Hence $\operatorname{tr}: R_{S} \rightarrow S_{S}$ is onto and so splits. Thus $S_{S}$ is a direct summand of $R_{S}$.

The conclusion concerning the Jacobson radical of $S$ follows from [2, Theorem 7.10, p. 132].

Proposition 1. A left $\Delta$ module is completely reducible as a $\Delta$ module if and only if it is completely reducible as an $R$ module. Moreover, a module is completely reducible as a left $R$-module if and only if it is completely reducible as an $S$ module.

Proof. A $\Delta$ module is annihilated by $J(\Delta)$ if and only if it is annihilated by $J(R)$ if and only if it is annihilated by $J(S)$. 
Proposition 2. Let $R$ be a left perfect ring and $G$ a completely outer group of automorphisms acting on $R$. Then $S$, the fixed ring of $R$ under $G$, and $\Delta$, the crossed product of $R$ with $G$ are left perfect.

Proof. Since $R$ is left perfect its Jacobson radical $J(R)$, is left $T$ nilpotent. So the Jacobson radical of $S, J(S)$, which is contained in $J(R)$ (by Lemma 2) is left $T$ nilpotent.

Also $S / J(S)$ is an Artinian ring since $S_{S}$ is a direct summand of $\boldsymbol{R}_{S}$. See Lemma 2 and [2, Proposition 7.3, p. 130]. Thus $S$ is a left perfect; hence, $S$ is semiperfect.

Now $R$ as a right $S$ module is finitely generated and projective; moreover, $\Delta$ is isomorphic to End $R_{S}[2$, p. 116]. Since $S$ is a direct summand of $R$, as a right $S$ module (Lemma 2) $R$ is an $S$ generator.

Let $e_{1}, \cdots, e_{n}$ be completely primitive idempotents orthogonal idempotents of $S$ such that $1=e_{1}+\cdots+e_{n}$. Furthermore, let $e_{1}, \cdots, e_{k}$ be a maximal family of mutually nonisomorphic idempotents. Then $\bar{R}=R / J(S) R$ is isomorphic, as a right $S$ module, to $\sum_{i=1}^{k}+\left(\bar{e}_{i} \cdot \bar{S}\right)^{m_{i}}$, where $\bar{e}_{i}=e_{i}+J(S), \bar{S}=S / J(S)$ and $m_{i}<\infty$, since $\bar{R}$ is finitely generated right $S$ module. Thus $R$ as a right $S$ module, is isomorphic to $\sum_{i=1}^{k} \oplus \sum_{i=1}^{m_{i}} \oplus P_{i j}$, where $P_{i j}$ is right $S$ isomorphic to $e_{i} S$, since idempotents can be lifted.

Let $f_{i j}$ be the projection of $R$ onto $P_{i j}$, then $f_{i j} \in$ End $R_{S}=\Delta$ and the $f_{i j}$ 's are orthogonal idempotents such that

$$
1=\sum_{i=1}^{k} \sum_{j=1}^{m_{i}} f_{i j} . \quad \text { Also } e_{i} S e_{i}=\operatorname{End}_{S}\left(e_{i} S\right)=\operatorname{End}_{S}\left(P_{i j}\right)=f_{i j} \Delta f_{i j}
$$

Since $e_{i} S e_{i}$ is a local ring, $f_{i j} \Delta f_{i j}$ is a local ring. Hence $f_{i j}$ is a completely primitive idempotent; therefore $\Delta$ is semiperfect.

We know that $\Delta$ modulo its Jacobson radical, $J(\Delta)$, is semisimple and idempotents can be lifted modulo $J(\Delta)$. Let $M$ be a left $\Delta$ module, by [1, Lemma 2.6, p. 473 ] in order that $M$ have a projective cover it suffices that for any left $\Delta$ module $B$ requiring no more generators than $M, B=J(\Delta) B$ implies $B=0$. But $B=J(\Delta) B=J(R) B$ and $R$ being left perfect implies $B=0$. Thus every left $\Delta$ module $M$ has a projective cover and $\Delta$ is then left perfect.

Let $T$ be an arbitrary left perfect ring and let $J(T)$ denote the Jacobson radical of $T$. Then for any nonzero left $T$ module $M, J(T) M$ is a proper submodule. See [1, p. 473]. Hence the natural map $\pi: M \rightarrow M / J(T) M$ is a minimal epimorphism.

Let $M$ and $N$ be left $T$ modules and $f$ a left $T$ epimorphism from $M$ to $N$. By $\bar{f}$, we mean the induced map from $M / J(T) M \rightarrow N / J(T) N$ given by $\bar{f}(m+J(T) M)=f(m)+J(T) N$ for $m \in M$. 
LEMMA 3. The following are equivalent:

(1) $f: M \rightarrow N$ is a minimal $T$ epimorphism.

(2) $\bar{f}: M / J(T) M \rightarrow N / J(T) N$ is an isomorphism. See [4, Proposition 8, p. 713].

Proposition 3. Let $M$ and $N$ be left $\Delta$ modules and $f$ a minimal $\Delta$ epimorphism form $M$ to $N$. Then $f$ is a minimal $R$ epimorphism and $f$ is a minimal $S$ epimorphism.

Proof. Certainly $f$ is an $R$ and an $S$ epimorphism. Since $J(\Delta) M=J(R) M=J(S) M$ and $J(\Delta) N=J(R) N=J(S) N$, then $\bar{f}$, which is a $\Delta$ isomorphism, is an $R$ and an $S$ isomorphism.

Proposition 4. Let $M$ be a left $\Delta$ module which is projective as an $S$ module, then $M$ is projective as a $\Delta$ module.

Proof. Let $P$ be the $\Delta$ cover of $M$ and $f: P \rightarrow M$ a minimal $\Delta$ epimorphism. Since $M$ is $S$ projective, $f$ splits as an $S$ epimorphism. Thus $P$ as an $S$ module is isomorphic to ker $f+X$, for some $S$ submodule $X$ of $P$. But $f$ is a minimal $S$ epimorphism (Proposition 3), therefore ker $f=0$. So $f$ is a $\Delta$ isomorphism and $M$ is $\Delta$ projective.

PROPOSITION 5. Let $M$ be a left $\Delta$ module which is projective as an $R$ module. Then $M$ is projective as a $\Delta$ module.

Proof. Let $P$ be the $\Delta$ projective cover of $M$ and $f$ a minimal $\Delta$ epimorphism. Now $f$ splits as an $R$ map, and $f$ is a minimal $R$ epimorphism, so $\operatorname{ker} f=0$.

Proposition 6. Let $M$ and $N$ be left $\Delta$ modules such that $M / J(\Delta) M$ and $N / J(\Delta) N$ are isomorphic as $R$ modules. If $M$ is $R$ projective there exists a $\Delta$ epimorphism $\phi: M \rightarrow N$. Moreover, if $N$ is $R$ projective, then $M$ and $N$ are $\Delta$ isomorphic. See [3, Lemma 5, p. 212].

Proof. We assume that $M$ and $N$ are completely reducible $\Delta$ modules. Hence they are completely reducible $R$-modules, by Proposition 1.

Now Nakayama in [3, p. 214] has shown that if $M$ and $N$ are isomorphic as $R$ modules, then they are isomorphic as $\Delta$ modules.

If $M$ and $N$ are arbitrary left $\Delta$ modules, then $M / J(\Delta) M$ and $N / J(\Delta) N$ are nonzero, completely reducible left $\Delta$ modules. We have 
assumed they are isomorphic as left $R$ modules; hence by the above argument, they are isomorphic as left $\Delta$ modules. Call the isomorphisms from $M / J(\Delta) M$ to $N / J(\Delta) N, f$.

Let $\pi: M \rightarrow M / J(\Delta) M$ and $\pi^{\prime}: N \rightarrow N / J(\Delta) N$ be the natural maps. Since $M$ is $R$ projective, it is $\Delta$ projective. Thus we can find a $\Delta$ homomorphism $g$ from $M$ to $N$ such that $\pi^{\prime} g=f \pi$. Now $g$ is an epimorphism since $\pi^{\prime}$ is a minimal $\Delta$ map.

If $N$ is also $R$ projective, it is $\Delta$ projective. Thus $g$ is an isomorphism.

Proposition 7. Let $M$ and $N$ be left $\Delta$ modules and $f a \Delta$ epimorphism from $M$ to $N$. If $f$ is a minimal epimorphism as an $R$ map, then $f$ is a minimal epimorphism as a $\Delta$ map. Furthermore, if $M$ and $N$ are $R$ projective, then $f$ is an isomorphism.

Proof. We know that $M / J(R) M$ and $N / J(R) N$ are isomorphic as $R$ modules and completely reducible. Hence they are isomorphic as $\Delta$ modules. Thus $f$ is a minimal $\Delta$ map.

If $M$ and $N$ are $R$ projective, then they are projective as $\Delta$ modules. Thus $f$ splits; let $g: N \rightarrow M$ be a $\Delta$ map such that $f g$ is the identity on $N$. Since the natural map $\pi: M \rightarrow M / J(\Delta) M$ is minimal $g$ is an isomorphism.

Proposition 8. Let $M, N$ be left $\Delta$ modules such that $M$ is a projective $R$ module. If $N$ is an $R$ direct summand of $M$, then $N$ is a $\Delta$ direct summand of $M$.

Proof. Since $M$ is $R$ projective and $N$ is a direct summand, then $N$ is $R$ projective. Hence $M$ and $N$ are $\Delta$ projective.

If $N$ is an $R$ direct summand of $M$, then $J(R) M \cap N=J(R) N$ so $J(\Delta) M \cap N=J(\Delta) N$. Thus $N / J(\Delta) N$ is a $\Delta$ submodule of the completely reducible $\Delta$ module $M / J(\Delta) M$.

Thus $N / J(\Delta) N$ is a $\Delta$ direct summand of $M / J(\Delta) M$, so there is a $\Delta$ epimorphism $\phi: M \rightarrow N / J(\Delta) N$. Let $\pi^{\prime}: N \rightarrow N / J(\Delta) N$, be the natural map. Since $M$ is $\Delta$ projective there exists a $\Delta$ map $\psi: M \rightarrow N$ such that $\pi^{\prime} \psi=\phi$. Now $\psi$ is an epimorphism since $\pi^{\prime}$ is minimal. Thus $\psi$ splits as a $\Delta$ map and $N$ is then a $\Delta$ direct summand of $M$.

Proposition 9. Let $M$ and $N$ be left $\Delta$ modules such that $N / J(R) N$ is an $R$ homomorphic image of $M / J(R) M$ and $M$ is projective as an $R$ module. Then $N$ is a $\Delta$ homomorphic image of $M$. Moreover, 
if $N$ is $R$ projective, then $N$ is a $\Delta$ direct summand of $M$.

Proof. Let $f$ be an $R$ epimorphism from $M / J(R) M$ to $N / J(R) N$. Since $R / J(R)$ is a semisimple, Artinian ring, $f$ splits; hence $N / J(R) N$ is a $R$ direct summand of $M / J(R) M$.

Now $G$ is a completely outer group of automorphisms of $R / J(R)$. The crossed product of $R / J(R)$ and $G$ is $\Delta / J(\Delta)$.

Applying Proposition 8 we see that $N / J(R) N$ is a $\Delta$ direct summand of $M / J(R) M$. Thus there is a $\Delta$ map $\phi$ from $M$ to $N / J(R) N$. Let $\pi^{\prime}: N \rightarrow N / J(R) N$ be the natural map. Since $M$ is $R$ projective, there is a $\Delta$ map $g: M \rightarrow N$ such that $\pi^{\prime} g=\phi$. Now $g$ is an epimorphism, since $\pi^{\prime}$ is a minimal $\Delta$ map.

If $N$ is $R$ projective, then $N$ is $\Delta$ projective. So $g$ splits.

Proposition 10. Let $g=|G|$, then $R^{g}$ is $\Delta$ isomorphic to $\Delta$.

Proof. $(R / J(R))^{g}$ is $R$ isomorphic to $\Delta / J(R) \Delta$.

Thus Proposition 6 implies $R^{8}$ and $\Delta$ are isomorphic.

Proposition 11. $R$ has a normal basis.

Proof. Proposition 10 imples $S^{8}$ is $S$ isomorphic to $R$ so $R$ has a normal basis by [2, Theorem 1.7, p. 118].

\section{REFERENCES}

1. H. Bass, Finitistic dimension and a homological generalization of semi-primary rings, Trans. Amer. Math. Soc., 95 (1960), 466-488.

2. Y. Miyashita, Finite outer Galois theory of non-commutative rings, J. Fac. Sci. Hokkaido Univ. Ser. 1, 19 (1966), 114-134.

3. T. Nakayama, Galois theory for general rings with minimal condition, J. Math. Soc. of Japan, 1 (1949), 203-216.

4. U. Skukla, On the projective cover of a module and related results, Pacific J. Math., 12 (1962), 709-717.

Received November 19, 1973.

UNIVERSITY OF CiNCINNATI 



\section{Pacific Journal of Mathematics}

\section{Vol. 56, No. $1 \quad$ November, 1975}

Shimshon A. Amitsur, Central embeddings in semi-simple rings .......... 1

David Marion Arnold and Charles Estep Murley, Abelian groups, A, such

that $\operatorname{Hom}(A,---)$ preserves direct sums of copies of $A \ldots \ldots \ldots .$.

Martin Bartelt, An integral representation for strictly continuous linear

operators ................................... 21

Richard G. Burton, Fractional elements in multiplicative lattices......... 35

James Alan Cochran, Growth estimates for the singular values of

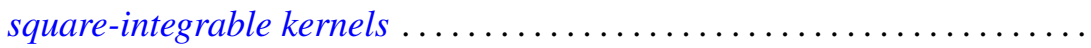

C. Martin Edwards and Peter John Stacey, On group algebras of central

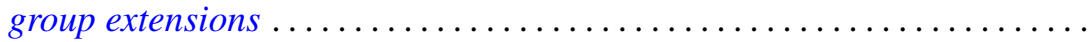

Peter Fletcher and Pei Liu, Topologies compatible with homeomorphism

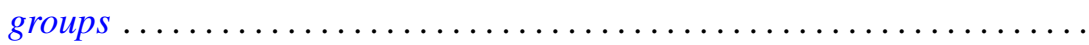

George Gasper, Jr., Products of terminating ${ }_{3} F_{2}(1)$ series ............ 87

Leon Gerber, The orthocentric simplex as an extreme simplex ............

Burrell Washington Helton, A product integral solution of a Riccati

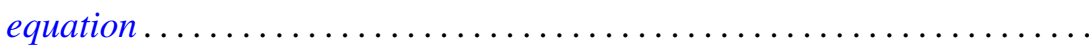

Melvyn W. Jeter, On the extremal elements of the convex cone of

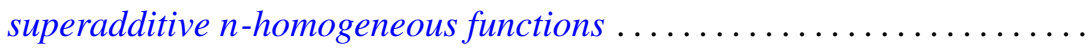

R. H. Johnson, Simple separable graphs .

Margaret Humm Kleinfeld, More on a generalization of commutative and

alternative rings. . .

A. Y. W. Lau, The boundary of a semilattice on an $n$-cell.

Robert F. Lax, The local rigidity of the moduli scheme for curves ...

Glenn Richard Luecke, A note on quasidiagonal and quasitriangular

operators .

Paul Milnes, On the extension of continuous and almost periodic functions

Hidegoro Nakano and Kazumi Nakano, Connector theory.

James Michael Osterburg, Completely outer Galois theory of perfect rings ..................................

Lavon Barry Page, Compact Hankel operators and the F. and M. Riesz

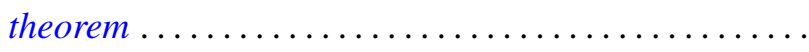

Joseph E. Quinn, Intermediate Riesz spaces..................... 225

Shlomo Vinner, Model-completeness in a first order language with a generalized quantifier.

Jorge Viola-Prioli, On absolutely torsion-free rings ..........

Philip William Walker, A note on differential equations with all solutions of integrable-square............................

Stephen Jeffrey Willson, Equivariant maps between representation 